\title{
Matrix Metalloproteinases Neutralizing Agents: A Possible Treatment Modality for Oral Lichen Planus (Preliminary Study)
}

Hossam Elsabagh

Alexandria University

Yasmine Gaweesh ( $\square$ yasmine.gaweesh@alexu.edu.eg )

Alexandria University

Jaylane Ghonima

Alexandria University

\section{Research Article}

Keywords: oral lichen planus, matrix metalloproteinases, MMP-9

Posted Date: February 2nd, 2021

DOI: https://doi.org/10.21203/rs.3.rs-152543/v1

License: (c) (i) This work is licensed under a Creative Commons Attribution 4.0 International License.

Read Full License 


\section{Abstract}

Background: Oral lichen planus is one of the most prevalent oral diseases. Matrix metalloproteinases have been implicated in its pathogenesis thus this study was executed to evaluate the effectiveness of matrix metalloproteinases neutralizing agents in the treatment of oral lichen planus.

Methods: Patients were assigned to either control group who received topical corticosteroids and antifungal treatment, or test group who received matrix metalloproteinases neutralizing spray. All patients were evaluated for disease severity and pain at baseline, 1 week, and 4 weeks after treatment.

Results: The mean pain score in test and control groups was 9.00 (0.89), and $9.33(0.82)$, respectively, at baseline while it was $2.33(1.75)$ and $2.83(1.72)$ at $4^{\text {th }}$ week follow up. The mean disease severity score was $4.33(0.52)$ for test group and $4.50(0.55)$ for control group at baseline and decreased to $1.83(0.75)$ and $2.17(0.98)$ at $4^{\text {th }}$ week follow up. Despite the better results seen in test group on the expense of control group, no statistically significant differences could be detected between groups at any timepoint.

Conclusion: The use of matrix metalloproteinases neutralizing agents might be an equally effective sole treatment for erosive oral lichen planus compared to the conventional treatment, without the risk of secondary candidiasis.

Trial registration: The study was registered retrospectively at ClinicalTrials.gov (NCT04336488), date $(07 / 04 / 2020)$

\section{Background}

Being one of the most commonly discussed and well-documented diseases affecting the oral mucosa, oral lichen planus (OLP) is enticing extensive research especially when it comes to its etiology, pathology and management. This chronic inflammatory, immunological and potentially malignant disease, with an estimated prevalence $5 \%$ worldwide, has six clinical patterns that occur individually or in combination: reticular, papular, plaque-like, ulcerative, erosive, and bullous.(1) Manifestations of these clinical forms range from totally asymptomatic keratotic lesions to very painful erosions and ulcerations.(2)

Symptomatic OLP treatment aims at relieving pain and improving patient's quality of life. A variety of treatment strategies have been proposed. These modalities includes corticosteroids, either in topical or systemic form,(3) also photochemotherapy.(4) In addition, cryosurgery, laser ablation and other drugs such as; azathioprine, laser, retinoids and selenium have been documented to be effective in recalcitrant cases.(5-7)

The exact etiology of OLP has not been fully explored yet. However, numerous factors have been implicated in triggering this immunological condition such as: genetic susceptibility, malnutrition, psychological stress, endocrine disorders and infectious agents. $(8,9)$. Oral lichen planus is basically a Tcell mediated inflammatory disease,(10) with many controversies about the mechanism of immune 
dysregulation in its pathogenesis. However, two main hypothesizes are widely accepted; Antigen-specific and non-specific mechanisms.(11) In antigen-specific pathway, basal keratinocytes exhibit antigenicity and these keratinocytes are destroyed by CD8 + cytotoxic T lymphocytes. While in non-specific mechanisms, the inflammatory environment in OLP lesions persists due to the degranulation of the mast cells and activation of the matrix metalloproteinase (MMPs).(12)

MMPs are a group of zinc-containing proteinases including at least 20 members, their main function is proteolytic degradation of the proteins in connective tissue matrices. Their function is regulated by a variety of endogenous inhibitors, mainly tissue inhibitors of metalloproteinases (TIMPs). Zhou et al in 2001 , (13) found that T cells from OLP lesions containing higher levels of MMP-9 in culture precipitates when compared to those obtained from peripheral blood of healthy controls. Furthermore, MMP-9 activators released from T cells induce the activation of pro MMP-9 causing the destruction of the epithelial basement membrane.(13) The destructed basement membrane in OLP lesions would not provide the signal essential for keratinocyte survival, thus provoking keratinocyte apoptosis. Furthermore, disruption of the basement membrane induced by MMP-9 might pave the way for antigen-specific CD $8+$ cytotoxic T-cells into the OLP epithelium, causing additional destruction.(12)

Whilst numerous studies have discussed the critical role played by MMPs in mediating mucosal destruction in OLP, no study has yet explored its actual significance on the clinical level. Therefore, the objective of this study was to compare the effectiveness of MMPs neutralizing agent in the treatment of OLP to that of corticosteroids and antifungal therapy.

\section{Material And Methods}

\section{Participants, materials and Study design:}

This pilot study being a part of an ongoing randomized controlled clinical trial (ClinicalTrials.gov (NCT04336488)), was conducted on 12 patients suffering from erosive OLP. Ethical approval was obtained from the ethics research committee at the Faculty of Dentistry, Alexandria University, Egypt (IRB 00010556) -(IORG 0008839). The study was performed in accordance with the modified Declaration of Helsinki's code for human clinical studies (2013). Informed consent was obtained from all participants after explaining the details of the study protocol.

Patients were recruited from the outpatient clinic of the Oral Medicine, Periodontology, Diagnosis, and Radiology Department, Faculty of Dentistry, Alexandria University, Egypt, during the period between December 2019 and January 2020.

The following inclusion criteria were applied: Patients with an age not less than 25 years, who were diagnosed with erosive OLP based on clinical examination and histopathological confirmation following the modified WHO criteria (2003) for the diagnosis of OLP(14). All patients had to be symptomatic and suffering from pain. Patients who were under anticoagulant medications, suffering from any systemic 
diseases or having any physical or mental abnormality, pregnant and lactating women, smokers, were excluded.

\section{Intervention and measurements:}

After detailed medical history taking, clinical examination, and biopsy taking, the Control group, comprised of 6 patients, was prescribed the treatment regimen of topical corticosteroid (triamcinolone acetonide $0.1 \%$ oral gel "Kenacort-A-Orabase) ${ }^{1}$ and topical anti-fungal agent (Miconaz oral gel) ${ }^{2}$, three times per day for 4 weeks. The test group included 6 patients given MMPs neutralizing agent (vaccinium macrocarp extract and vaccinium mytillus, Orochem spray $20 \mathrm{ml})^{3}$, three times per day for 4 weeks.(15)

Clinical changes from baseline were evaluated at 2 time points: 1 week and 4 weeks after commencement of the treatment, using the two following measurements as primary and secondary outcomes;

1- Subjective pain and discomfort severity were recorded using the Numeric Rating Scale (NRS) (16), categorized to (No pain (NRS 0), Mild pain (NRS 1-3), Moderate pain (NRS 4-7), Unbearable pain (NRS 810)). Patients were asked to assign a numerical score on the scale verbally to rate their pain intensity and the number was recorded. $(17,18)$

2- Objective disease severity using (19) Thongprasom scoring system (this scoring system was selected despite the availability of more valid scoring systems to allow comparison of the results with those of other clinical trials as it is the most commonly used to date)(20), categorized to; score 5 was assigned to a lesion of white striae with erosive areas $>1 \mathrm{~cm}^{2}$, score 4 assigned to a lesion of white striae and erosive areas $<1 \mathrm{~cm}^{2}$, score 3 assigned to a lesion of white striae and atrophic areas $>1 \mathrm{~cm}^{2}$, score 2 assigned to a lesion of white striae and atrophic areas $<1 \mathrm{~cm}^{2}$, score 1 assigned to a lesion having only white striae, and score 0 assigned to normal mucosa. Photographical records of the lesions were taken at baseline, after 1 , and 4 weeks.

\section{Randomization, blinding and allocation concealment}

Patients were randomized after providing informed consent either into a test group or control by unpredictable random sequence using simple randomization procedures (computerized random numbers). Additionally, patients were blinded as to which group they were assigned. The allocation list was concealed by sequentially numbered, opaque, sealed envelopes.

\section{Sample size estimation}

For the original randomized clinical trial, A sample size of 18 patients per group (number of group $=2$ ) (total sample size $=36$ patients) is the enough required sample to detect a standardized effect size of 
0.6150 (minimum difference in mean pain scores using NRS) change in primary outcome $(21,22)$, as statistically significant with $80 \%$ power and at a significance level of $95 \%$ (accepted $a$ of 0.05 ).

Sample size was increased to 40 to control for attrition bias.(23) The sample size was calculated using GPower version 3.1.9.2.(24) Thirty percent (12/40 patients) of the original sample size was considered suitable to conduct this pilot study ( $n=12,6$ patients per group).

\section{Statistical analysis}

Differences in NRS and disease severity scores were compared using Mann Whitney $U$ test. Whereas, changes across time were assessed using Friedman test. $P$ value was adjusted for multiplicity by multiplying the uncorrected $p$-value by the number of comparisons made to set a significance level at 0.05.(25) Data were analyzed using IBM SPSS software package version 25.0. (Armonk, NY: IBM Corp)

\section{Results}

From the patients recruited for an ongoing clinical trial, twelve were enrolled in this pilot study. The mean (SD) age of patients was 49.00 (7.64) in test group and 54.50 (8.31) in control group. No significant differences between groups were observed regarding the age or gender distribution (Table 1).

Table 1

Demographics:

\begin{tabular}{|llll|}
\hline & \multicolumn{1}{c}{$\begin{array}{l}\text { Test } \\
(\mathbf{n = 6})\end{array}$} & $\begin{array}{l}\text { Control } \\
(\mathbf{n = 6})\end{array}$ \\
\hline Age: Mean (SD) & $49.00(7.64)$ & $54.50(8.31)$ \\
\hline Gender: $\mathrm{n}(\%)$ & Females & $3(50 \%)$ & $5(83.3 \%)$ \\
\cline { 2 - 4 } & Males & $3(50 \%)$ & $1(16.7 \%)$ \\
\hline
\end{tabular}

The mean NRS pain score presented in Table 2 showed statistically significant reduction from baseline to first week ( $P$-value $=0.006 /<0.0001)$, as well as from baseline to 4 th week $(P$-value $=<0.0001 /<0.0001)$ in both test and control groups respectively. However, no statistically significant difference was observed between the two groups at any time point. Categorical pain severity distribution among the patient's groups are demonstrated in Table 3 
Table 2

NRS results among patients in the test and control:




Table 3

Categorical pain severity distribution among patients in the test and control:

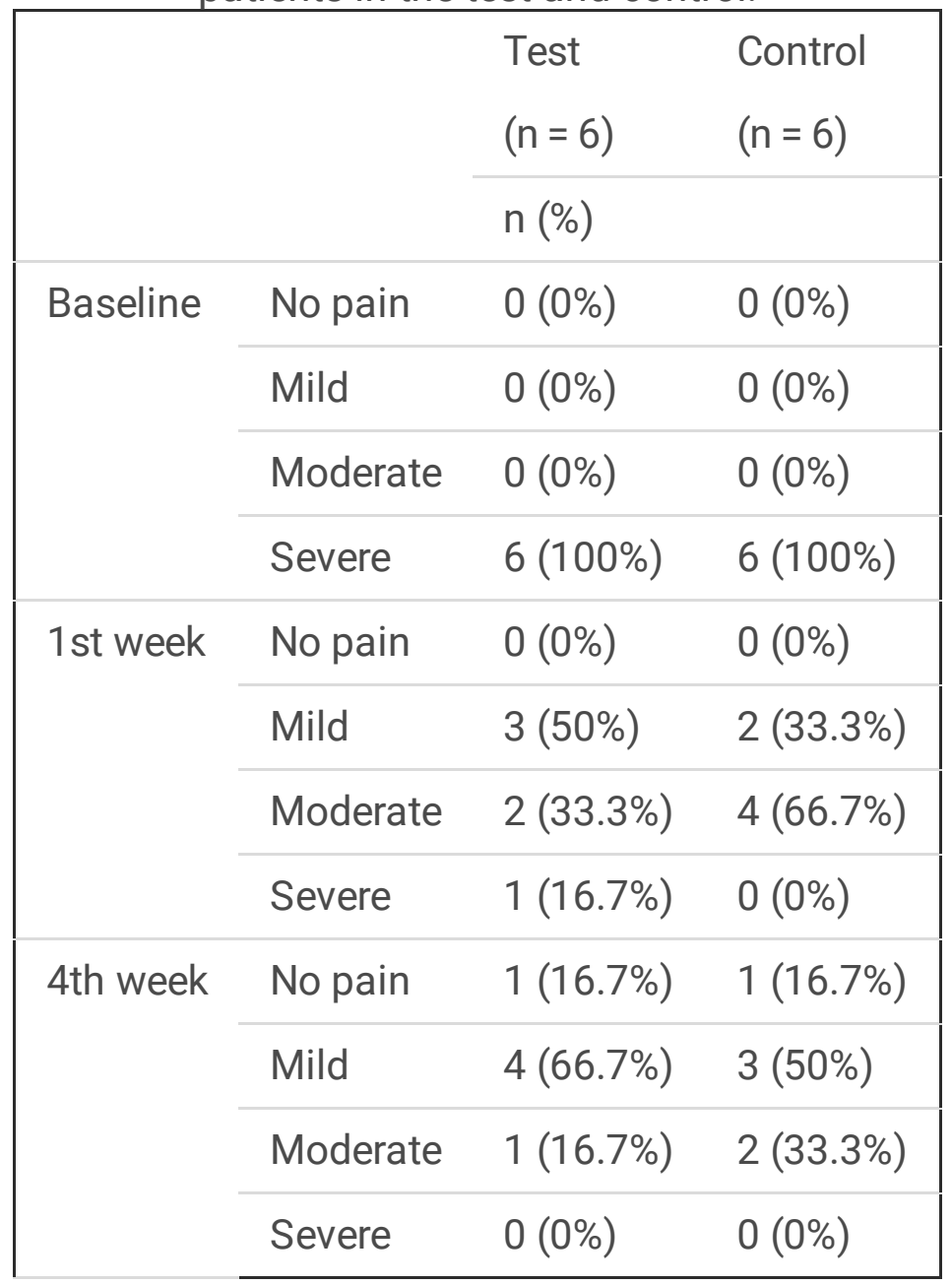

Regarding the disease severity score, a clinically valuable reduction in the mean score was observed from baseline to 4th week in both groups where the mean Thongprasom score was $4.33(0.52)$ and $4.50(0.55)$ at baseline in the test and control groups, respectively, while it was $1.83(0.75)$, and $2.17(0.98)$ at 4th week although this reduction did not reach statistical significance. Noteworthy, the mean Thongprasom score was slightly higher in the control group as compared to the test group even though this difference was not statistically significant. (Table 4). 
Table 4

Thongprasom scores among patients in the test and control:

\begin{tabular}{|llll|}
\hline & $\begin{array}{l}\text { Test } \\
(\mathbf{n}=6)\end{array}$ & \multicolumn{1}{c}{$\begin{array}{l}\text { Control } \\
(\mathbf{n}=6)\end{array}$} & \\
\cline { 2 - 4 } & Mean (SD) & \\
\hline Baseline & $4.33(0.52)$ & $4.50(0.55)$ & 0.599 \\
\hline 1 st week & $3.00(1.27)$ & $2.67(0.82)$ & 0.599 \\
\hline 4th week & $1.83(0.75)$ & $2.17(0.98)$ & 0.525 \\
\hline$p$ value & $P_{1}=0.114, P_{2}=0.102$ & $P_{1}=0.069, P_{2}=0.114$ \\
\hline$P_{1}$ : differences between baseline and 1st week & \\
\hline$P_{2}$ : differences between baseline and 4th week & \\
\hline
\end{tabular}

\section{Discussion}

There is a continuous diligent search for alternative treatment modalities for OLP due to the absence of a definite cure. An optimum therapy would be one with adequate efficacy and minimal side effects. The selection of the treatment modality tested in this study was based on a deep insight into the pathogenesis of OLP. The expression of MMPs in OLP has been documented in literature since the nineties of the last decade.(26) Furthermore, the integral role of MMP-9 in the pathogenesis of OLP has been proven in multiple studies. $(12,13)$ Sugerman et al. suggested that epithelial basement membrane disruption, one of the main events in OLP pathogenesis, is mainly executed by T-cell secreted MMP-9.(12) Moreover, MMP-2 and MMP-3 were expressed in the lesional epithelium of OLP.(13)

Hence it was postulated that neutralizing the effect of MMPs might hamper major events taking place in the pathogenesis of OLP and, thereafter, induce improvement in the clinical manifestations of the disease.(27) Up to our knowledge, no MMPs inhibiting or neutralizing factors have been tested in the treatment of OLP to date.

Shrivastava et al. identified certain plant tannins and procyanidin (PCD) fractions that are capable of binding to MMPs.(28) In a later study, the same authors discovered that the association of two of these PCDs resulted in $84 \%$ ( $\pm 9.2 \%)$ reduction in the activity of MMPs $2,3,9$, and $24 .(15)$ These PCDs constituted the main active ingredients of the drug under investigation.

Besides, the formulation incorporated glycerol resulting in an osmotically active hypertonic solution that allows exudation of a hypotonic fluid in the erosive and ulcerative lesions disinfecting them.(15) Beneficial effect of the hypertonicity of the formulation was reflected in the lack of the need to prescribe a 
topical antifungal agent along with it. Noteworthy, prescription of a topical antifungal is indispensable when topical corticosteroids are administered where secondary candidiasis is reported to be the most common side effect.(29)

The application of the solution, being applied as a spray, was found to be much easier and more pleasant than the commonly used adhesive paste formulation used for topical corticosteroids. Patients commonly complain of the difficulty of applying adhesive pastes on the soft palate or near the pillars of the fauces. In addition, the unpleasant feel of the adhesive paste is another common complain. Such complains where absent with the administration of the spray formulation. A similar conclusion was withdrawn in a study by Hegarty et al. in 2002.(30)

Preliminary results represented in this pilot study have shown significant improvements in pain scores in both the test and control groups. In the 4th week follow up visit no patients presented with severe pain in both groups. Only $16.7 \%$ presented with moderate pain in the test group versus $33.3 \%$ in the control group at the last follow up. Disease severity scores significantly improved between baseline and last follow up visit in both groups although this improvement did not reach statistical significance.

Other topical non-immunosuppressant agents were tested in previous studies. Mansourian et al. (31) compared the effect Aloe Vera mouthwash to that of triamcinolone acetonide $0.1 \%$ paste in the treatment of OLP and found no significant differences between the 2 groups in both pain and disease severity scores. Thongprasom score was found to $1.39 \pm 0.16$ in the Aloe Vera group after 4 weeks which is in accordance with the present study where the mean score was found to be $1.83(0.75)$.

Qataya et al. (6) investigated the effectiveness of topical selenium as compared to conventional treatment and systemic selenium in the management of OLP. The mean Thonprasom score after 6 weeks was $2.273 \pm 1.27$ as compared to $1.83(0.75)$ in the present study while NRS score was reported to be 3.5 \pm 1.97 while it was $2.33(1.75)$ in the current study at the same timepoints. The difference in the NRS scores between the two studies could be referred to the different mechanisms of action of the used therapeutic agents.

Despite the promising results of the current preliminary study, several limitations could be defined. Primarily, the small sample size is a definite drawback in the study affecting the study power. However, study results only represent the preliminary results of an ongoing registered randomized clinical trial. Another limitation is the short follow up period included in the study. Other studies with longer follow up are recommended to investigate the long-term effects of the drug formulation tested and possible side effects that may emerge. Finally, investigations working on formulating a specific MMP-9 inhibiting agent are strongly recommended. Such a formulation would be more potent than the drug tested in the current study being a merely neutralizing agent and would target the specific MMP proven to be involved in the pathogenesis of OLP.

\section{Conclusions}


Based on the results of this preliminary study, it could be concluded that the use of MMPs neutralizing agents in the management of erosive form of OLP might be equally effective sole treatment in the management of erosive OLP to the conventional treatment improving both pain and disease manifestations, without the increased risk of secondary candidiasis.

\section{Abbreviations}

OLP: oral lichen planus

MMPs: matrix metalloproteinases

TIMPs: tissue inhibitors of metalloproteinases

NRS: numeric rating scale

\section{Declarations}

\section{- Ethics approval and consent to participate}

Ethical approval was obtained from the ethics research committee at the Faculty of Dentistry, Alexandria University, Egypt (IRB 00010556) -(IORG 0008839). Informed consent was obtained from all participants after explaining the details of the study protocol.

\section{- Consent for publication}

Not applicable

\section{- Availability of data and materials}

The datasets used and/or analysed during the current study are available from the corresponding author on reasonable request.

\section{- Competing interests}

The authors declare that they have no competing interests

- Funding

The study was funded by the authors

- Author's contributions 
Author 1 (co-author): Hossam H Elsabagh contributed to conception, design, data acquisition and interpretation, drafted and critically revised the manuscript.

Author 2 (corresponding author): Yasmine Y Gaweesh contributed to conception, design, data interpretation, drafted and critically revised the manuscript.

Author 3 (co-author): Jaylane K Ghonima contributed to study design, data interpretation and analysis and critically revised the manuscript.

\section{- Acknowledgements}

The authors would like to express their gratitude to the staff of Oral Medicine, Periodontology, Oral Diagnosis and Oral Radiology department of Alexandria University. To Dr Marwa Gebril BDS, MSc. of Oral Medicine, for her great help in recruiting the patients, to Dr Hams Hamed BDS, MSc. Assistant lecturer of dental public health, faculty of dentistry, Alexandria university, Egypt. for her great help in data statistical analysis

\section{References}

1. Cheng YS, Gould A, Kurago Z, Fantasia J, Muller S. Diagnosis of oral lichen planus: a position paper of the American Academy of Oral and Maxillofacial Pathology. Oral Surg Oral Med Oral Pathol Oral Radiol. 2016;122(3):332-54.

2. Payeras MR, Cherubini K, Figueiredo MA, Salum FG. Oral lichen planus: focus on etiopathogenesis. Archives of oral biology. 2013;58(9):1057-69.

3. Kaplan I, Ventura-Sharabi Y, Gal G, Calderon S, Anavi Y. The dynamics of oral lichen planus: a retrospective clinicopathological study. Head and neck pathology. 2012;6(2):178-83.

4. Maloth KN, Velpula N, Kodangal S, Sangmesh M, Vellamchetla K, Ugrappa S, et al. Photodynamic therapy-a non-invasive treatment modality for precancerous lesions. Journal of lasers in medical sciences. 2016;7(1):30.

5. Gupta S, Ghosh S, Gupta S. Interventions for the management of oral lichen planus: a review of the conventional and novel therapies. Oral Dis. 2017.

6. Qataya PO, Elsayed NM, Elguindy NM, Ahmed Hafiz M, Samy WM. Selenium: A sole treatment for erosive oral lichen planus (Randomized controlled clinical trial). Oral Diseases.n/a(n/a).

7. Mutafchieva MZ, Draganova-Filipova MN, Zagorchev PI, Tomov GT. Effects of Low Level Laser Therapy on Erosive-atrophic Oral Lichen Planus. 2018;60(3):417.

8. Bombeccari GP, Guzzi G, Tettamanti M, Giannì AB, Baj A, Pallotti F, et al. Oral lichen planus and malignant transformation: a longitudinal cohort study. Oral Surgery, Oral Medicine, Oral Pathology, Oral Radiology, and Endodontology. 2011;112(3):328-34.

9. Nosratzehi T. Oral Lichen Planus: an Overview of Potential Risk Factors, Biomarkers and Treatments. Asian Pac J Cancer Prev. 2018;19(5):1161-7. 
10. Thornhill MH. PLENARY ABSTRACT: The Current Understanding of the Aetiology of Oral Lichen Planus. Oral Diseases. 2010;16(6):507-8.

11. Roopashree MR, Gondhalekar RV, Shashikanth MC, George J, Thippeswamy SH, Shukla A. Pathogenesis of oral lichen planus - a review. Journal of Oral Pathology \& Medicine. 2010;39(10):729-34.

12. Sugerman PB, Savage NW, Walsh LJ, Zhao ZZ, Zhou XJ, Khan A, et al. The pathogenesis of oral lichen planus. Critical reviews in oral biology and medicine : an official publication of the American Association of Oral Biologists. 2002;13(4):350-65.

13. Zhou XJ, Sugerman PB, Savage NW, Walsh LJ. Matrix metalloproteinases and their inhibitors in oral lichen planus. Journal of cutaneous pathology. 2001;28(2):72-82.

14. van der Meij EH, Schepman KP, van der Waal I. The possible premalignant character of oral lichen planus and oral lichenoid lesions: a prospective study. Oral Surg Oral Med Oral Pathol Oral Radiol Endod. 2003;96(2):164-71.

15. Shrivastava R, Deshmukh S. A New Therapeutic Approach to Treat Oral Mucositis Using Specific MMP Blockers in an Osmotically Active Solution. Journal of Cancer Research and Treatment. 2013;1(1):4-11.

16. Paice JA, Cohen FL. Validity of a verbally administered numeric rating scale to measure cancer pain intensity. Cancer nursing. 1997;20(2):88-93.

17. Elsabagh HH, Moussa E, Mahmoud SA, Elsaka RO, Abdelrahman H. Efficacy of Melatonin in prevention of radiation-induced oral mucositis: A randomized clinical trial. Oral Dis. 2020;26(3):56672.

18. Boonstra AM, Stewart RE, Koke AJ, Oosterwijk RF, Swaan JL, Schreurs KM, et al. Cut-Off Points for Mild, Moderate, and Severe Pain on the Numeric Rating Scale for Pain in Patients with Chronic Musculoskeletal Pain: Variability and Influence of Sex and Catastrophizing. Front Psychol. 2016;7:1466.

19. Thongprasom $K$, Luengvisut $P$, Wongwatanakij $A$, Boonjatturus $C$. Clinical evaluation in treatment of oral lichen planus with topical fluocinolone acetonide: a 2-year follow-up. Journal of oral pathology \& medicine. 2003;32(6):315-22.

20. Elsabagh HH, Gaweesh Y, Ghonima J, Gebril M. A novel comprehensive scoring system for oral lichen planus: A validity, diagnostic accuracy, and clinical sensitivity study. Oral Surgery, Oral Medicine, Oral Pathology and Oral Radiology. 2020.

21. Daniel WW, Cross CL. Biostatistics: a foundation for analysis in the health sciences: Wiley; 2018.

22. Killeen PR. An alternative to null-hypothesis significance tests. Psychological science. 2005;16(5):345-53.

23. Pannucci CJ, Wilkins EG. Identifying and avoiding bias in research. Plastic and reconstructive surgery. 2010;126(2):619.

24. Faul F, Erdfelder E, Lang A-G, Buchner A. G* Power 3: A flexible statistical power analysis program for the social, behavioral, and biomedical sciences. Behavior research methods. 2007;39(2):175-91. 
25. Chen S-Y, Feng Z, Yi X. A general introduction to adjustment for multiple comparisons. J Thorac Dis. 2017;9(6):1725-9.

26. Sutinen $M$, Kainulainen T, Hurskainen T, Vesterlund E, Alexander J, Overall C, et al. Expression of matrix metalloproteinases (MMP-1 and-2) and their inhibitors (TIMP-1,-2 and-3) in oral lichen planus, dysplasia, squamous cell carcinoma and lymph node metastasis. Br J Cancer. 1998;77(12):2239-45.

27. Lavanya N, Jayanthi P, Rao UK, Ranganathan K. Oral lichen planus: An update on pathogenesis and treatment. Journal of oral and maxillofacial pathology: JOMFP. 2011;15(2):127.

28. Shrivastava R. Clinical evidence to demonstrate that simultaneous growth of epithelial and fibroblast cells is essential for deep wound healing. diabetes research and clinical practice. 2011;92(1):92-9.

29. Thongprasom K, Dhanuthai K. Steriods in the treatment of lichen planus: a review. Journal of oral science. 2008;50(4):377-85.

30. Hegarty A, Hodgson T, Lewsey J, Porter S. Fluticasone propionate spray and betamethasone sodium phosphate mouthrinse: a randomized crossover study for the treatment of symptomatic oral lichen planus. Journal of the American Academy of Dermatology. 2002;47(2):271-9.

31. Mansourian A, Saheb-Jamee M, Momen-Beitollahi J, Momen-Heravi F, Esfehani M, Khalilzadeh O. Comparison of aloe vera mouthwash with triamcinolone acetonide $0.1 \%$ on oral lichen planus: a randomized double-blinded clinical trial. The American journal of the medical sciences. 2011;342(6):447-51. 